$\xi=-1$

\title{
Verification and power consumption measurement of 'eid signal in eddy stone beacon
}

\author{
Ki-bong Kim ${ }^{1 *}$, Ki-mun Keum ${ }^{2}$, Chang-bok Jang ${ }^{2}$, Oh-Seok Kwon ${ }^{3}$ \\ ${ }^{1}$ Dept. Computer Information Processing, Daejeon Health Institute of Technology, Chungjeongno 21, Dong-gu, Daejeon, 34504 Krea \\ ${ }^{2}$ R2soft co.Ltd., IT Venture Town 405, Techno-9ro, Yuseong-gu, Daejeon, 34027 Korea \\ ${ }^{3}$ Dept. Computer Engineering, Chungnam National University, Daehak-ro 99, Yuseong-gu, Daejeon, 34134 Korea \\ *Corresponding authorE-mail: kbkim@hit.ac.kr
}

\begin{abstract}
Background/Objectives: Recently, users are receiving various services through smart phones. Especially, with the emergence of IoT, the service by collecting information from things is expanding, and various techniques for using BLE beacons are being studied.

Methods/Statistical analysis: Services that use BLE beacons periodically transmit Bluetooth signals and mobile devices receive signals from near beacons. In this case, the mobile application should also periodically scan for the Bluetooth signal. It consumes additional power from the smartphone. Therefore, low power consumption is an important issue for services that use beacons. Optimized results should be found by measuring power at various levels of beacon scan period and beacon advertising period.

Findings: As a result of this research, the power consumption exponentially increases as the scan period becomes shorter, but the beacon recognition rate does not improve significantly. The average power consumption was $9.6 \%$ at $10 \mathrm{~s}$ Scan Period, but $6.5 \%$ at $30 \mathrm{~s}$ and $5.6 \%$ at $100 \mathrm{~s}$. Advertising Period affected the change of Beacon Recognition Rate though the effect of power consumption was small. The Beacon Recognition Rate was very low when the Advertising Period was short, but it was $50 \%$ to $70 \%$ at $200 \mathrm{~ms}$ to $1000 \mathrm{~ms}$. In the service used in this study, the scan period was set to 30 seconds and the advertisement period to $1000 \mathrm{~ms}$ for optimization.

Improvements/Applications: Applying the results of this paper to various beacons and services requires additional experimental design that takes into account beacons and other indicators that affect variables, such as scan execution time, signal strength, and beacon life.
\end{abstract}

Keywords: BLE Beacon; Eddy Stone; 'Eid; Beacon Scan Period; Beacon Advertising Period

\section{Introduction}

Beacon Bluetooth technology can create an environment that can provide users with a variety of active services. Recently, enhanced Bluetooth technology, BLE (Bluetooth Low Energy) ${ }^{1}$, has been activated and the beacon functionality and platform has been upgraded to include more options for the systems you need.

Smartphones are not only owned by many people, but also have features that enable them to use the service anytime and anywhere. Therefore, developing a mobile application that receives beacon Bluetooth signals enables location-based services ${ }^{2}$ to be performed to users moving near beacons. That is, the beacon has a spatial characteristic that it can apply a zone corresponding to a radius of several tens of meters compared with the proximity distance of the NFC, and corresponds only to a narrow area compared to a very wide area service of GPS. This user location identification can be used for product sales, store advertising, online coupon delivery, informational notices, unattended services, and provides an automated and convenient user experience.

Despite these advantages, however, the weakness of security due to the nature of wireless signals exposed to the outside world is hindering the growth of the beacon market. [1], [3] The iBeacon ${ }^{4}$ platform's Bluetooth signal is well suited for services that may be exposed to anyone outside of the beacon, because all users near the beacon can receive signals, but there was a disadvantage in that it was difficult to provide services that needed to be secured. The Eddystone ${ }^{5}$ platform was able to escape this problem by providing EID (Ephemeral ID) [5] signals, giving the Bluetooth signal a cryptographic effect and a transient effect. The EID signal uses a cryptographic algorithm to send the signal in a random string and provides security because the signal changes over time. Another issue to note is beacons consume power from mobile devices. The beacon transmits the Bluetooth signal to the outside every certain cycle, which is called 'Advertising'. The beacon must send the signal continuously, so the power consumption should be minimized. The BLE beacon is a new one that has reduced its power consumption and has greatly contributed to the activation of the beacon service market. However, a smartphone receiving a beacon signal still needs to be scanned ${ }^{6}$ and consumes power from the device. Sometimes, some of service may require that the smartphone continuously scan for the presence of a beacon signal, in which case the power consumption of the smartphone will be a major concern to the user.

In this paper, the discussion points are limited to the following environments. The beacon type uses only BLE beacons, and the Bluetooth signal uses Eddystone-EID signal to provide security. In Chapter 2, we refer to previous research and related studies. In Chapter 3, we measure the power according to beacon scan period and beacon advertisement period and derive the test result. The conclusions are presented in Chapter 4. 


\section{Related works}

\subsection{Beacon characteristic}

In this paper beacons have two states: Advertising mode and Connection mode. The Advertising mode is a normal state of a beacon, and it periodically transmits UUID $^{7}$ value and signal strength information in the beacon to external space. Peripherals capable of receiving Bluetooth signals can distinguish each beacon through the UUID value and estimate the distance from the approximate beacon through the signal strength. If there are multiple beacons, you can also use triangulation ${ }^{2,8}$ to determine the location of mobile device users. The Advertising mode uses the GAP(Generic Access Profile $)^{9}$ protocol and corresponds to the 'Service Processing' stage which performs necessary functions through beacons. In order to use a beacon, beacon must exist in the Advertising state by default.

There are UID, URL, TLM, and EID frames ${ }^{5}$ in the Eddystone Advertising mode signal and each signal type is encoded in the upper 4 bits of the first octet of the Eddystone frame. Each signal has a value of $0 \times 00,0 \times 10,0 \times 20,0 \times 30$. Eddystone-UID, which is commonly used for simple identification of beacons, consists of 16 bytes, which is the same as UUID, and has 10-byte Namespace and 6-byte Instance components. The Eddystone-URL uses a compressed format URL structure that can be used directly on the client side. Eddystone-TLM is a signal with various status data of beacons. The Eddystone-EID, which provides encryption attributes, consists of 10 bytes. The overall Eddystone frame format is shown in Table 1.

Table 1: Eddy stone Frame Format [10]

\begin{tabular}{|c|c|c|c|}
\hline $\begin{array}{l}\text { Byte } \\
\text { offset }\end{array}$ & Value & Description & Data Type \\
\hline 0 & $0 \times 02$ & Length & $\begin{array}{l}\text { Flags. CSS v5, Part A, } \S \\
1.3\end{array}$ \\
\hline 1 & $0 \times 01$ & Flags data type value & \\
\hline 2 & $0 \times 06$ & Flags data & \\
\hline 3 & $0 \times 03$ & Length & $\begin{array}{l}\text { Complete list of 16-bit } \\
\text { Service UUIDs. Ibid. } \S \\
1.1\end{array}$ \\
\hline 4 & $0 \times 03$ & $\begin{array}{l}\text { Complete list of 16-bit } \\
\text { Service UUIDs data type } \\
\text { value }\end{array}$ & \\
\hline 5 & 0xAA & 16-bit Eddystone UUID & \\
\hline 6 & $0 x F E$ & - & \\
\hline 7 & $0 x ?$ & Length & $\begin{array}{l}\text { Service Data. Ibid. } \S \\
1.11\end{array}$ \\
\hline 8 & $0 \times 16$ & $\begin{array}{l}\text { Service Data data type } \\
\text { value }\end{array}$ & \\
\hline 9 & 0xAA & 16-bit Eddystone UUID & \\
\hline 10 & $0 x F E$ & - & \\
\hline $11 \sim 31$ & - & $\begin{array}{l}\text { Eddystone Frame } 20 \text { Bytes } \\
\text { (UID, URL, TLM, EID) }\end{array}$ & \\
\hline
\end{tabular}

The Connection Mode is connected One-on-One to mobile device for internal value setting of beacon, and can change various information including UUID. The Connection mode uses the GATT(Generic Attribute Profile) ${ }^{11}$ protocol, and Eddystone defines 'Eddystone Configuration GATT Service'12 to support this.

Eddystone Configuration GATT Service contains various beacon properties. 'Capabilities' have slot information such as the maximum number of slots that can be transmitted simultaneously. 'Active Slot' supports data read / write of slot. 'Advertising Interval' determines the time interval during which advertising is conducted periodically. 'Radio Tx Power' can indicate and change the advertising signal strength. 'Lock State' and 'Unlock' are responsible for locking and unlocking when you access the beacon. 'EID Identity Key' handle EID settings and key management. 'Factory Reset' performs beacon slot initialization. In order to use Connection Mode, Unlock must be performed before beacon connection. If password is specified through Lock State, user must enter the correct password before connection is performed.

\subsection{Eddystone EID}

Eddy stone-EID can provide security, but it needs a Google Cloud Server because it needs to be associated with Google that developed Eddystone. The beacon's private key and public key information should also be used. First, the beacon must be registered with the Google Cloud Server, so the beacon registration request is executed. In this process, the beacon can generate the 128-bit Identify Key using the private key and public key information of beacon, the 'Rotation Period Exponent' value, and the 'Time Counter' value. Rotation Period Exponent is an integer value that can be assigned arbitrarily and is used for the purpose of making a unique influence on the generation of Identify Key. Time Counter is a time value that can not be set to a specific value and is used to implement a random seed. The Google Cloud Server can also generate the same 128-bit Identify Key using the private key and public key information of server, the Rotation Period Exponent value, and the Time Counter value, and verifies these Identify Key values match each other, and confirm that a correct EID signal can be generated from the beacon.

Once the beacon is authenticated for EID generation, it can now send out a valid EID signal. The mobile device around the beacon runs Bluetooth scan through the installed mobile application, receives this EID signal and sends it to the Google Cloud Server. The server can identify the beacon signal by checking the EID signal, and thus can return a string such as the promised UUID key to the mobile application. This string value allows the application to determine and provide the appropriate service to the user. This process is illustrated in Figure 1. The system of Figure 1 is the "R2-Etizone Service" to be used as a object of experiment in this paper, that is a service that receives a beacon signal and automatically causes the mobile device to perform public etiquette such as vibration, brightness, and camera. The implementation of the system is not covered in this paper.

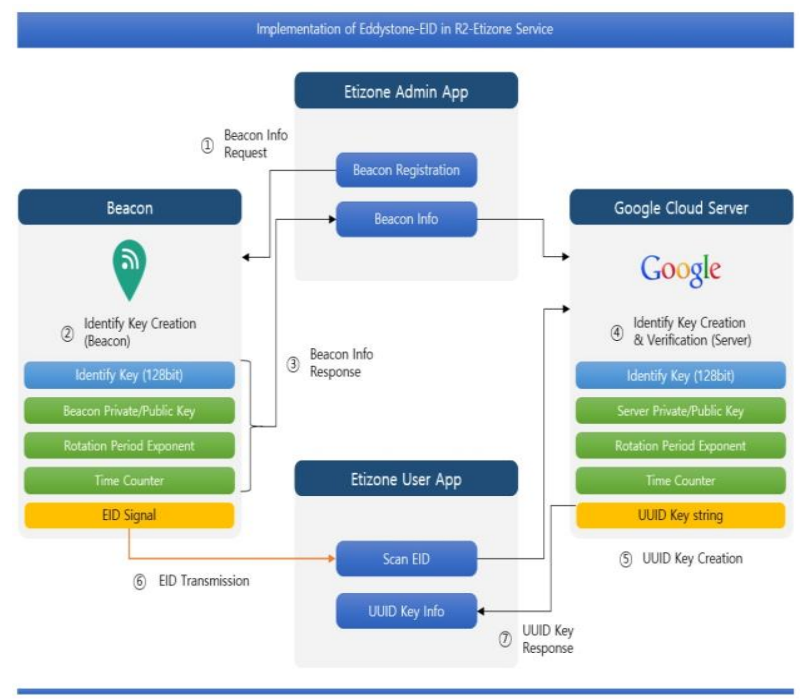

Fig. 1: Example of Implementation of Eddy stone-EID.

\section{Tests and results}

\subsection{Test variable description}

In this test, to find a way to minimize the power consumption of the smartphone when using EID by controlling the existing variables for the result of implementing the signal transmission of Eddystone-EID. The important variables that should be recognized in the test are as follows.

- Power consumption: The difference between the amount of power consumed during the designated time of idle states that did not run the test service and the amount of power consumed during the designated time after running the test service. This is an important concern in the test. 
- Beacon Recognition Rate: Percentage that indicates whether the mobile application is able to recognize the Bluetooth signal each time from nearby beacon when it is scanned. 'Scan Period' and 'Advertising Period' is involved in this variable. In a well-designed system, Beacon Recognition Rate is less affected by other variables, but nonetheless, in this test, the Beacon Recognition Rate is measured and the results are examined.

- Application Reactivity: Indication of whether the mobile application can quickly recognize the presence of beacon Bluetooth signals in the vicinity and provide a better response to the user. 'Scan Period' is involved in this variable. In this paper, Application Reactivity is not measured.

- Scan Run-time: It means the operating time of the SCAN when the smartphone confirms whether there is a surrounding signal for receiving the Bluetooth signal. Normally, the time is set to about 5 seconds. If you increase the Scan Runtime, you can improve the Beacon Recognition Rate, but it consumes more power of the smartphone. Conversely, if you reduce the Scan Run-time, the Beacon Recognition Rate may decrease, but the power of the smartphone can be saved even more. In this paper, this variable is fixed at the reference value.

- Scan Period: It means a time interval when the smartphone periodically checks whether there is a surrounding signal for receiving a Bluetooth signal. Reducing the Scan Period to perform more frequent scans can improve Application Reactivity and Beacon Recognition Rate, but consumes more power on the smartphone. Conversely, if you extend the Scan Period to reduce the number of scans, the Application Reactivity may decrease, but the power of the smartphone can be saved even more.

- Advertising Period: When a beacon transmits a Bluetooth signal, it means the time interval between the previous transmission and the next. It is usually short, less than 1 second. Advertisement cycle is an important variable that can dramatically change Beacon Recognition Rate. The Advertising Period generally has no significant impact on the power of the smartphone, therefore, this paper will focus on the result of beacon recognition rate.

\subsection{Test procedure and condition}

The dependent variable we want to check in this test is the power consumption and we will additionally check the Beacon Recognition Rate. The Beacon Recognition Rate may depending on the type of service being performed. In this experiment, this figure was determined to be $70 \%$. The independent variables of the test are the Scan Period and the Advertising Period. The Scan Runtime is fixed to 5 seconds. The detailed conditions for testing are shown in Table 2.

Table 2: Other Detailed Conditions Used in the Power Consumption Test

\begin{tabular}{ll}
\hline Test conditions & Values \\
\hline Battery remaining & $100 \%$ \\
Screen brightness & minimum \\
Display settings & Screen always on \\
Lock screen & Off \\
App running status & background \\
Number of beacons & 2 \\
Power measurement time & 1 hour \\
\hline
\end{tabular}

The Scan Period change test is set to $10 \mathrm{~s}, 20 \mathrm{~s}, 30 \mathrm{~s}, 50 \mathrm{~s}$, and $100 \mathrm{~s}$, and the Advertising Period is fixed to $500 \mathrm{~ms}$. The Advertising Period change test is set to $100 \mathrm{~ms}, 200 \mathrm{~ms}, 300 \mathrm{~ms}, 500 \mathrm{~ms}$, and $1000 \mathrm{~ms}$, and the Scan Period is set to $10 \mathrm{~s}, 20 \mathrm{~s}, 30 \mathrm{~s}, 50 \mathrm{~s}$, and $100 \mathrm{~s}$. In both tests, the power measurement confirms the results after eight hour and will compare the power measurement results of idle state with no application installed. The experimental model is Samsung Galaxy S7, and the battery capacity is $3000 \mathrm{mAh}$. Estimote Location Beacon was used for BLE beacon. The power measuring instrument used was Monsoon Power Monitor FTA22D, and the analysis software was PowerTool 4.0.4.12.

\subsection{Test result and discussion}

The experimental results of the Scan Period change test are shown in Figure 2. The power consumption decreased as the Scan Period became longer, especially when the Scan Period was shorter. The average power consumption was $9.6 \%$ at 10 s Scan Period, but $6.5 \%$ at $30 \mathrm{~s}$ and $5.6 \%$ at $100 \mathrm{~s}$. Therefore, the shorter the Scan Period is, the worse the power efficiency becomes.

It has been confirmed that the scan period has a sufficient effect on the speed of signal recognition. Beacon signals were normally received at $73.7 \%$ at a $10 \mathrm{~s}$ Scan Period, $71.3 \%$ at 30 s, and $48.9 \%$ at $100 \mathrm{~s}$. The optimized state in this service is the lowest power consumption with a Beacon Recognition Rate of over $70 \%$, the Scan Period of 30 seconds will be selected in the graph in Figure 2 .

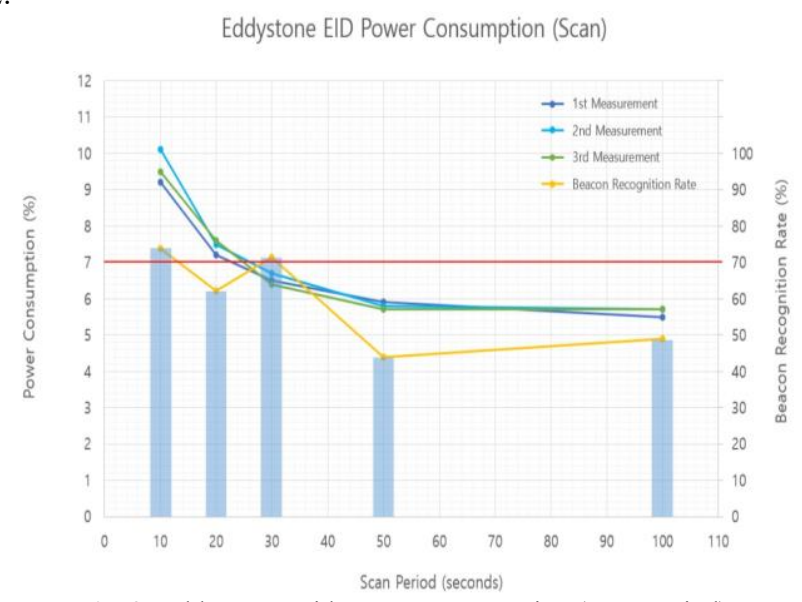

Fig. 2: Eddy Stone Eid Power Consumption (Scan Period)

The experiment results for the Advertising Period change test are shown in Figure 3. Changing the Advertising Period did not significantly affect power consumption. Consumed power in smartphone devices $6.7 \%$ at $100 \mathrm{~ms}$ Advertising Period, $6.5 \%$ at $300 \mathrm{~ms}$, and $6.3 \%$ at $1000 \mathrm{~ms}$. Therefore, the Advertising Period will not be a significant factor in considering the devices power state. The Advertising Period will be excluded from the factor for power state optimization.

The impact of changes in the Advertising Period was important to Beacon Recognition Rate. When the Advertising Period was very short, $100 \mathrm{~ms}$, the value was very low because the system could not receive it normally. From the time the Advertising Period was set to $200 \mathrm{~ms}$, the recognition rate was $50 \%$ to $70 \%$, and the longer the Advertising Period, the more the Beacon Recognition Rate tended to increase. Choosing an optimized Advertising Period is a reasonable choice for $1000 \mathrm{~ms}$ when considering the power consumed by the beacon. However, since the Scan Run-time is usually set to about 5 seconds, a long period of several seconds will cause in improper results.

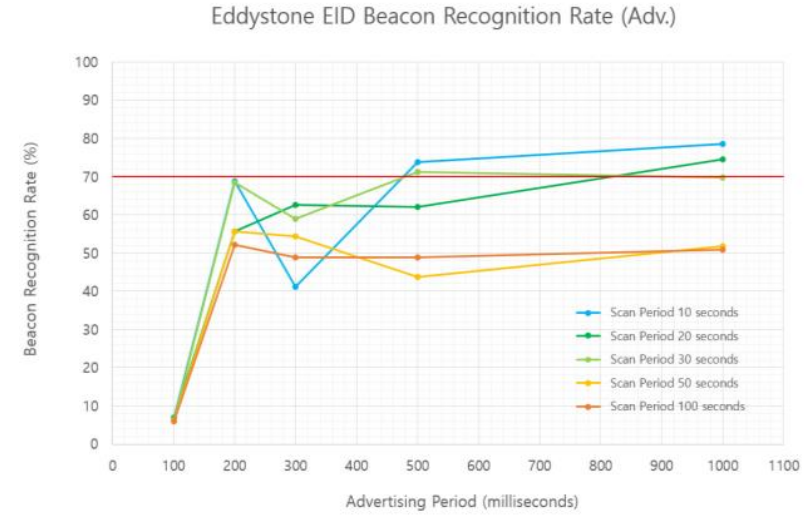

Fig. 3: Eddy Stone Eid Power Consumption (Advertising Period). 


\section{Conclusion}

The purpose of this paper is to find the Scan Period and the Advertising Period which can consume the power of the user's smartphone to the minimum when the service using the Eddystone-EID signal of the BLE beacon and the mobile application is performed. And examined the Beacon Recognition Rate according to the Advertising Period to find the optimized number. The power consumption of the smartphone is a major concern when the user uses the service, and the Beacon Recognition Rate can determine the service quality. To minimize power consumption and maintain beacon recognition above a certain level, each element must find a compromise that does not adversely affect other elements.

As a result of this study, as the Scan Period was shortened, the power consumption increased due to the exponential relationship and the benefit of Beacon Recognition Rate was not significant. Advertising Period did not affect power consumption, but there was an effect on Beacon Recognition Rate change. For the optimized results, it was advantageous to determine the Scan Period to be 30 seconds and the Advertising Period to be $1000 \mathrm{~ms}$. In order to apply the results of this paper to various beacons and services, additional experimental design considering beacon and application affecting variables will be necessary.

\section{Acknowledgment}

This work was supported by research fund of Chungnam National University.

\section{References}

[1] Carles G, Joaquim O, Josep P, Overview and Evaluation of Bluetooth Low Energy: An Emerging Low-Power Wireless Technology. Sensors, 2012, pp. 11734-11753.

[2] Sudarshan S C, Beacon Placement for Indoor Localization using Bluetooth. Intelligent Transportation Systems, 2008, 10.1109/ITSC.2008.4732690.

[3] John P, John B, Mayank B, Marcel H, Rhonda S, Lily C, Karen S, Guide to Bluetooth Security. National Institute of Standards and Technology, 2017, 10.6028/NIST.SP.800-121r2.

[4] Nic N, Apple iBeacon technology briefing. Journal of Direct, Data and Digital Marketing Practice, 2014, pp. 222-225.

[5] https://developers.google.com/beacons/eddystone, Google Beacon Platform, 2017.

[6] Naeim A, Ashish B, Edward C, Bluetooth and Wi-Fi MAC Address Based Crowd Data Collection and Monitoring: Benefits, Challenges and Enhancement. Australasian Transport Research Forum 2013 Proceedings, 2013, pp. 1-17.

[7] Leach P, Mealling M, Salz R, A Universally Unique IDentifier (UUID) URN Namespace. Request for Comments 4122, 2005, pp. $1-32$.

[8] Lee H C, Lee D M, A Study on Localization System using 3D Triangulation Algorithm based on Dynamic Allocation of Beacon Node. The Journal of Korean Institute of Communications and Information Sciences, 2011, pp. 378-385.

[9] https://www.bluetooth.com/ko-kr/specifications/assignednumbers/generic-access-profile, Bluetooth SIG, 2017.

[10] https:/github.com/google/eddystone/blob/master/ protocolspecification.md, GitHub, 2017.

[11] https://www.bluetooth.com/specifications/gatt/generic-attributesoverview, Bluetooth SIG, 2017.

[12] https://github.com/google/eddystone/tree/master/configurationservice, GitHub, 2017. 